\title{
Politique, histoire et histoire scolaire
}

Le cas de la République tchèque

\section{Petr Čornej}

Traducteur : Carole Sormanek

\section{OpenEdition}

\section{Journals}

Édition électronique

URL : http://journals.openedition.org/ries/3249

DOI : $10.4000 /$ ries.3249

ISSN : 2261-4265

\section{Éditeur}

Centre international d'études pédagogiques

\section{Édition imprimée}

Date de publication : 1 mars 1997

Pagination : 81-92

ISSN : 1254-4590

\section{Référence électronique}

Petr Čornej, « Politique, histoire et histoire scolaire », Revue internationale d'éducation de Sèvres [En ligne], 13 | 1997, mis en ligne le 24 juillet 2013, consulté le 03 mai 2019. URL : http:// journals.openedition.org/ries/3249 ; DOI : 10.4000/ries.3249 


\section{Politique, histoire et histoire scolaire}

\section{Le cas de la République tchèque ${ }^{1}$ \\ Petr Čornej}

Les événements qui, en novembre 1989, ont entraîné la chute du régime communiste ont logiquement bouleversé le paysage historiographique tchèque. Parallèlement, l'enseignement alors en vigueur a été profondément remanié et cette nouvelle conception s'est imposée dans tous les établissements scolaires, des écoles primaires aux écoles supérieures. Partout, des voix se sont élevées pour exiger un récit non idéologisé du passé, un récit qui mettrait un terme à la manipulation de l'histoire, aussi bien chez les scientifiques que dans le corps enseignant et raviverait la mémoire historique. La nouvelle devise exprima la nécessité de combler rapidement les «pages blanches » (c'est-à-dire délibérément ignorées par le régime communiste) de l'histoire locale et mondiale, de rectifier les interprétations dénaturées et de forger une véritable conscience historique. Tâche complexe et ardue qui, comprise dans toute son acception, ne peut réussir, car une société libre est formée d'une constellation d'opinions personnelles et de points de vue différents (liés à la couleur politique) sur le passé. Les premiers jours de liberté ont ainsi vu émerger des débats historiques chargés d'émotion qui tendaient à réévaluer l'histoire en fonction de choix individuels quelque peu précipités et proposant une rapide réorganisation du paysage politique. Ils trouvèrent une tribune dans les journaux, les magazines et les médias audiovisuels. Le refus de mêler le domaine politique au récit du passé finit par aboutir à la tendance inverse. Ces deux inclinations antagonistes, qui pourtant s'influencent et s'imprègnent mutuellement, sont l'émanation directe de la complexité des circonstances dans lesquelles le processus de rupture avec le régime communiste s'est déroulé.

Aucune autre évolution n'était possible, car la recherche de nouvelles lectures de l'histoire prit place dans l'effervescence des discussions consacrées au sens des réformes politico-économiques, à la notion de politique intérieure et extérieure et à l'existence même de l'État. Dès avant le printemps 1990, un violent conflit éclata entre les représentations tchèque et slovaque sur l'organisation institutionnelle. Trois ans plus tard, la partition - pacifique - de la République tchécoslovaque (dont l'existence remontait, excepté la parenthèse de la Seconde Guerre mondiale, à 1918) était consommée et, le $1^{\text {er }}$ janvier 1993 , deux nouveaux États indépendants furent constitués : la République tchèque et la République slovaque. Les arguments historiques trouvaient un écho dans tous

1 Cet article a été traduit par Carole Sormanek. 
les débats politiques sérieux, auxquels des historiens professionnels (un nouveau type d'historiens fit son apparition : l'historien-commentateur des faits politiques) participaient fréquemment. L'histoire et les historiens jouaient donc un rôle (qu'ils jouent encore aujourd'hui) dans les discussions politiques concernant l'actualité brûlante.

L'effondrement du système communiste, qui imposait une vision de l'histoire unique et "obligatoire" au service de ses propres intérêts, induisit dans l'esprit de beaucoup de gens une confusion qui s'étendit à l'enseignement de cette matière dispensé dans les écoles primaires et secondaires. Le régime communiste considérait en effet que la tâche de l'histoire scolaire était de raconter "la légitimité de l'évolution de la société humaine" (dans un esprit marxiste, naturellement) et de développer "la conviction communiste et la conscience politique des élèves ${ }^{2} »$. Bien que des différences sensibles, selon les écoles et la personnalité des professeurs, fussent à relever entre les objectifs déclarés et les réalisations pratiques de l'enseignement, l'état général n'avait rien de réjouissant. Certes, les écoles primaires et secondaires dispensaient aux élèves une importante somme de connaissances factuelles, mais on leur transmettait l'histoire de façon partiale. Le problème de l'enseignement de l'histoire ne se réduit évidemment pas à la pression politique ; il est dû également à la forte féminisation du corps enseignant, par ailleurs soumis à nombre d'obligations sans rapport direct avec l'enseignement. De plus (pourquoi ne pas le reconnaître ?) la conception marxiste de l'histoire, superficielle, simple et facile à appréhender, n'exigeait aucun effort de réflexion particulier et convenait à beaucoup de professeurs, qui s'y conformaient et étaient incapables d'envisager une conception différente.

Après les changements politiques, ils eurent les pires difficultés à abandonner leurs schémas immuables et à accepter d'autres conceptions et interprétations, qui les déstabilisaient et les déboussolaient. Il n'empêche que la majorité des enseignants accueillit le nouveau régime avec enthousiasme et suivit spontanément les cours que les historiens des écoles supérieures et des instituts scientifiques leur dispensaient. Dans ces conditions, il est heureux que lesdites institutions, qui s'enrichirent, après la révolution, de penseurs indépendants venus de la dissidence et de représentants des jeunes générations, fussent restées en contact avec le monde occidental et eussent gardé, du moins dans le cercle restreint des spécialistes, une notion de la voie empruntée par l'historiographie. Le processus de rupture avec les stéréotypes si profondément enracinés n'en fut pas moins douloureux pour nombre de professeurs du primaire et du secondaire, comme en témoignent les efforts acharnés qu'ils déployèrent pour que soit donné à la relation du passé un cadre bien fixe et politiquement arrêté auquel se référer. N’oublions pas de mentionner le fait que certains enseignants

2 Programme scolaire des collèges et lycées, Prague, 1983, page 3. 
adoptèrent la démarche de nombre de médias, qui consistait à tenter de résoudre les problèmes du passé en se contentant de prendre à contre-pied l'interprétation communiste, dénuée de toute objectivité. Le danger d'une nouvelle perception sans nuance de l'histoire fut une menace réelle des premières années de l'après-révolution, mais s'affaiblit avec le temps. Les enseignants de la plus jeune génération, c'est-à-dire ceux qui arrivèrent en fin de cursus après 1990 et qui furent formés pour exercer en toute indépendance, n'ont plus ces problèmes. Ce qui ne veut pas dire que les liens entre la politique et l'histoire scolaire aient été rompus.

\section{Rééquilibrer le passé}

Le lien unissant domaine politique et enseignement de l'histoire s'exprime sans aucun doute le plus nettement dans le récit de l'histoire récente, à savoir de la période couvrant les années 1918 à 1989 ; néanmoins, les interprétations communistes ont touché l'histoire dans son ensemble. Tandis que beaucoup de gens considèrent que les fameuses "pages blanches » à combler se rapportent quasi exclusivement à l'histoire $\mathrm{du} \mathrm{xx}^{\mathrm{e}}$ siècle, les milieux scientifiques et scolaires pensent que toutes les époques historiques sont concernées, bien qu'à des degrés différents.

La préhistoire, l'Antiquité et, en partie, l'histoire du Moyen Age ne furent en effet pas exposées à une pression idéologique aussi massive de la part du régime communiste. Quoique, là également, les travaux scientifiques officiels - et les manuels scolaires davantage encore - étaient dominés par la conception marxiste orthodoxe, qui perçoit l'histoire comme une succession légitime de formations socio-économiques et qui explique leur dynamique par la lutte permanente que se livrent les classes sociales antagonistes. Le fait de considérer la problématique dans son seul aspect économico-social relégua au second plan le domaine de la culture spirituelle, réduite à une simple superstructure plus ou moins dépendante de l'état de la base économique. Ce modèle s'appliquait prioritairement à la présentation de l'histoire moderne, que le régime communiste tenait absolument à contrôler, car l'objectif visé, la «désinterprétation » du passé récent, faisait partie des moyens qu'il mettait en œuvre pour asseoir sa légitimité. Il était donc logique que le changement politique et social de novembre 1989 mette au jour la nécessité d'une révision non seulement du récit de l'histoire contemporaine, mais également de toute l'histoire, c'est-à-dire de l'histoire tchèque et mondiale depuis la préhistoire jusqu'à nos jours.

Aujourd'hui, les livres et les enseignants ne limitent plus leur présentation de la préhistoire à la seule explication de la genèse de l'homme et de la société humaine, mais familiarisent les élèves avec les diverses grandes théories. La conception de l'histoire de l'Antiquité a elle aussi changé. Elle ne s'appuie plus uniquement sur l'antagonisme citoyens/esclaves, mais prend également en compte le champ d'action complexe de la société d'alors et tente de saisir la 
signification de la civilisation antique pour la culture du Moyen Age et des temps modernes.

On observe un décalage peut-être encore plus important dans l'interprétation de l'histoire moyenâgeuse. L'accent qui était mis auparavant sur l'histoire économique et politique a fait place à un intérêt pour la vie quotidienne locale de l'Europe médiévale; en effet, les acquis fondamentaux des médiévistes mondiaux qui nous ont précédés (entre autres, Jacques Le Goff, Georges Duby, Emmanuel Le Roy-Ladurie, Arno Borst, Karl Bosl, Ferdinand Seibt, Aron Gurevič) sont désormais couramment exposés dans les manuels scolaires, ainsi que les résultats les plus remarquables des scientifiques tchèques (parmi lesquels Josef Macek, František Šmahel, Josef Válka), qui ne purent être publiés que de façon fort confidentielle au cours des dernières décennies communistes.

Cela eut pour conséquence la modification de la perception de l'histoire tchèque antérieure. L'explication traditionnelle, née au XIXe siècle et revue par les marxistes, qui insistait surtout sur le rôle de l'État tchèque du Moyen Age et sur les efforts de la population urbaine et rurale, fut révisé en profondeur. Les nouveaux textes mentionnent l'importance de l'action (jusque-là omise) de la noblesse dans le processus d'édification et de fonctionnement de l'organisation étatique, ainsi que l'apport pour la civilisation de la chrétienté et de l'Église catholique. L'histoire religieuse, récemment encore délibérément négligée, est ainsi peu à peu intégrée à l'historiographie, et ce à tous les niveaux scolaires.

On peut dire qu'aujourd'hui les présentations tchèque et européenne de l'histoire moyenâgeuse, de ses origines à la charnière du XIVe siècle, ne sont plus fondamentalement différentes. En revanche, on voit apparaître des points de frottement entre les interprétations historiques tchèque et européenne du XVe au XVII siècle. Longtemps l'idée dominante (émise après 1848 par František Palacký, fondateur de l'historiographie tchèque moderne, confortée par l'autorité du premier président tchécoslovaque, T. G. Masaryk, et dûment remodelée par les marxistes) consista à porter sur la Réforme tchèque du Xve siècle (Jan Hus et le hussitisme) et sur les grandes réformes européennes qui lui succédèrent un jugement en tous points positifs, et ce quel que ce soit l'angle d'observation adopté pour les relater : confessionnel, national, social ou purement « de classes ». Le lien ainsi établi entre les forces réformatrices et les tendances démocratisantes et de progrès n'ont jamais satisfait les historiens catholiques tchèques, qui ont toujours, ouvertement ou officieusement, mis en doute la perception si profondément ancrée qui faisait du hussitisme l'époque la plus glorieuse de l'histoire tchèque. Les catholiques profitèrent de la libéralisation politique qui suivit novembre 1989 pour remettre en question la contribution de la période hussite et réformatrice et pour mettre en valeur l'ère de la contre-réforme victorieuse et du baroque, aux XVI et XVIII siècles, péjorativement qualifiées par les protestants, les libéraux et la gauche politique de temno (ténèbres). Pourtant, la vision catholique n'eut pas un écho très favorable dans la société tchèque, qui se méfie des raisonnements de cette confes- 
sion et qui soupçonne l'Église romaine de n'aspirer qu'au pouvoir et à l'enrichissement. Heureusement, l'histoire scolaire actuelle a retenu le jugement lucide et sensé des dernières générations de chercheurs. Celles-ci se gardent des jugements extrêmes et tentent d'appréhender l'histoire tchèque du $\mathrm{XV}^{\mathrm{e}}$ au XVII ${ }^{\mathrm{e}}$ siècle d'un point de vue rigoureusement historique, c'est-à-dire en évitant les anachronismes et la dramatisation, et en donnant une image aussi équilibrée que possible de cette époque sensible ${ }^{3}$. On redonne ainsi à la Réforme, à la Contre-Réforme, à la culture baroque et au travail des Églises évangéliques la place et le rôle qui leur reviennent. Ce qui n'empêche pas la polémique entre l'Église catholique romaine et les Églises protestantes de continuer, par publications interposées, bien que les deux parties ne jurent que par l'œcuménisme, mot d'ordre à la mode.

Les discussions concernant la relation et la valeur à accorder à l'époque de la "renaissance nationale», ainsi qu'on appelle traditionnellement ici le processus de constitution de la nation tchèque moderne, sont encore plus vives. Il date de la fin du XVIII ${ }^{\mathrm{e}}$ siècle et connut son apogée au cours de la seconde moitié du XIXe siècle. A ce propos, il convient de préciser qu'on donne au substantif "nation" en Europe centrale un sens différent de celui que les régions situées à l'ouest du Rhin ou en Amérique du Nord lui reconnaissent. Tandis que l'Europe occidentale et le continent nord-américain ont peu à peu fait de la "nation » une notion politique (les habitants et, plus tard, les citoyens d'un État donné définissent la nation), l'Europe centrale (et orientale) lui attribue un caractère typiquement ethnique : ce qui détermine la nation, ce sont la langue et la culture communes à ses ressortissants ${ }^{4}$.

La "nation tchèque » (au sens ethnique du terme) vécut pendant plusieurs siècles au sein de la monarchie multinationale habsbourgeoise, où l'allemand s'imposa, à partir de la seconde moitié du XVIII e siècle, comme langue administrative et où la population germanophone occupait pratiquement tous les postes politiques importants. Dans ce contexte de formation de la "nation tchèque moderne ", la lutte pour la reconnaissance des droits civiques et politiques passait logiquement par la lutte pour l'égalité culturelle et linguistique. Il en allait de même pour toutes les autres ethnies très minoritaires - qu'elles aient appartenu à l'Empire habsbourgeois, russe, ottoman ou allemand -, qui vivaient très mal le fait de ne pas jouir de l'égalité des droits politiques. Les efforts politiques et culturels de la nation tchèque aboutirent à la naissance d'une république indépendante, en octobre 1918 : la République tchécoslovaque. Ce nouvel État représentait, pour les Tchèques et pour certains Slovaques, la concrétisation de leurs

3 Le livre de Vít VInas intitulé Jan Nepomucký, česká legenda (Jan Nepomucký - une légende tchèque), Prague, 1993, en est un exemple typique.

4 Parmi les historiens tchèques, Jiří Kořálka a donné de la conception politique et ethnique de la nation l'analyse la plus pertinente dans Co je národ? (Qu'est-ce qu'une nation?), Prague, 1969, page 8-9, 13-17 ; voir également Tschechen im Habsburgerreich und in Europa 1815-1914, München-Wien, 1991, p. 23-25. 
idéaux ; cependant, les minorités ethniques (principalement allemande et hongroise), traumatisées par le brusque changement de leur situation, ne purent s'accoutumer à cette nouvelle donne et appelèrent de leurs vœux le rattachement de leur territoire qui à l'Allemagne, qui à la Hongrie, contribuant ainsi à la disparition de la Tchécoslovaquie en 1938-1939. Après la Seconde Guerre mondiale, la constitution d'une nouvelle République tchécoslovaque ne put être envisagée que sur la base du respect du principe ethnique. La conférence de Potsdam ne s'opposa pas au transfert de la plus grande partie des Allemands et des Hongrois (l'expatriation de ces derniers fut très rapidement stoppée) hors du territoire tchécoslovaque.

Cette petite digression, forcément schématique, devrait permettre de faire comprendre dans quelle mesure le sentiment d'incertitude qui entourait l'existence de l'État et de la nation tchèque, sis entre l'Allemagne et la Russie, sentiment renforcé par l'occupation allemande entre 1939 et 1945 et par l'intervention de l'armée soviétique en 1968, influence la perception de l'histoire. Par conséquent, il n'est pas surprenant que la majorité des Tchèques et des historiens tchèques considèrent la «renaissance nationale " et la république indépendante de l'entre-deux-guerres comme des étapes essentielles de leur histoire.

Paradoxalement, ces sentiments provoquèrent l'émergence d'avis qui ne jettent pas sur la renaissance tchèque et la naissance de la Tchécoslovaquie indépendante un regard systématiquement positif. Ainsi, dans les milieux dissidents de la Tchécoslovaquie de l'après-août (1968) s'exprima l'opinion selon laquelle la fin de la monarchie habsbourgeoise multinationale (multiethnique), en 1918, et la formation de petits États sur les ruines de cet empire ne firent pas le bonheur de l'Europe centrale, car le pouvoir ainsi laissé vacant ouvrit la porte à l'expansionnisme allemand (nazi) et russe (communiste). C'est ainsi qu'on en vint à critiquer la «renaissance nationale», à qui on reprochait d'avoir perturbé, par une analyse partiale de la question, la coexistence pacifique des ethnies allemande et tchèque sur le territoire tchèque, d'avoir fait obstacle au développement de la société civique et d'avoir participé à la mise en œuvre de processus dont les conséquences se font sentir encore aujourd'hui en Europe centrale et entravent l'entrée de la République tchèque dans les communautés euro-atlantiques. La Tchécoslovaquie de l'entre-deux-guerres porte également sa part de responsabilité dans cette évolution, pour n'avoir pas su régler de manière conséquente la question des nationalités et pour avoir été très réticente et réservée vis-à-vis des revendications des Allemands des Sudètes, signant ainsi sa propre condamnation ${ }^{5}$.

5 Le plus systématique de ces avis est celui de Podiven (pseudonyme collectif du juriste Petr Pithart, de l'historien Milan Otáhal et du psychiatre Petr Přihoda), Čě̌i v dějinách nové doby (Les Tchèques dans I'histoire moderne), Prague, 1991 (ainsi que les publications samizdats antérieures). La revue Dějiny et současnost se fit d'ailleurs l'écho du débat critique autour de ce livre. Karel Kučera exprima de sérieuses réserves à propos de cette publication dans Historie \& historici, Prague, 1992, p. 219-233. 
Ces critiques déclenchèrent dès avant 1989, et davantage encore après, des discussions houleuses qui virent la majorité des spécialistes de l'histoire du $\mathrm{XIX}^{\mathrm{e}}$ et $\mathrm{du} \mathrm{XX}^{\mathrm{e}}$ siècle rejeter autant les jugements des chantres nostalgiques de la monarchie habsbourgeoise ${ }^{6}$ que les avis purement moralisateurs de certains dissidents se réclamant de l'autorité du philosophe Jan Patocka, qui évaluent le passé à la lumière de la conception actuelle des droits civiques et des libertés individuelles et font abstraction du contexte historique dans lequel la constitution de la nation tchèque moderne vit le jour ${ }^{7}$. Malgré ces affrontements, que les pages des périodiques et les écrans de télévision ont rendus publics, le concept de "renaissance nationale " continue d'être traité, dans les manuels de toutes les écoles, comme une étape marquante de l'histoire tchèque, mais elle est désormais dépouillée de l'idéalisation antérieure et de toute diabolisation du rôle historique de l'État habsbourgeois ${ }^{8}$.

La conception dominante de l'histoire de la Tchécoslovaquie des années 1918 à 1938 est étroitement liée à cette interprétation. Les historiens actuels pensent dans leur grande majorité que cet État n'était pas seulement la réalisation des aspirations politiques du peuple tchèque et slovaque d'alors, mais que cette république, malgré toutes ses difficultés, ses insuffisances et ses échecs, était aussi la concrétisation de l'idée moderne d'une société civique et démocratique. Sa disparition, en 1938-1939, et l'impossibilité, à la fin de la Seconde Guerre mondiale, de la reconstituer dans sa forme originelle sont, d'après eux, la conséquence de l'expansionnisme des régimes totalitaires, du régime nazi tout d'abord, du régime soviétique ensuite. Il va sans dire que cette analyse ne correspond pas à l'interprétation communiste qui, d'un côté, accordait un certain prix à la naissance de la Tchécoslovaquie indépendante et, de l'autre, faisait tout pour la dévaloriser. Dans cette optique, on qualifiait la république de l'entre-deux-guerres d'État bourgeois (en d'autres termes, capitaliste), on dénonçait l'absence de justice sociale et on désavouait ses dirigeants.

L'interprétation que les textes scolaires tchèques actuels donnent de la période comprise entre 1939 et 1989 dénonce les régimes totalitaires (fascistes et communistes) et parle des crimes commis et tus par le système communiste

6 Je pense ici en particulier à Středni Evropa (Europe centrale), revue samizdat jusqu'en 1989, officielle ensuite.

7 Je fais allusion, entre autres, aux propos tenus par l'historien Emanuel Mandler et le politologue Bohumil Doležal.

8 En plus du travail de Jiři Korálka (voir note $n^{\circ} 4$ ), plusieurs livres sur cette problématique sont parus ces dernières années. Les plus importants sont: Jiři Rak, Byvali Čechové. České historické mýty a stereotypy (Les Anciens Tchèques. Mythes et stéréotypes historiques tchèques), Prague, 1994 ; Vladimír Macura, Znameni zrodu. České národní obrozeni jako kulturni typ (Les Signes d'une naissance. La Renaissance tchèque, une caractéristique culturelle), 2e éd., Prague, 1995 ; Zdenek Hojda - Jiři Pokorný, Pomniky a zapomníky (Monuments de mémoire et monuments d'oubli), Prague, 1996. 
au niveau local et mondial ${ }^{9}$. La présentation de la Seconde Guerre mondiale a également été modifiée. La reconnaissance exclusive du rôle de l'Union soviétique a cédé la place à une description (parfois trop) détaillée de l'action des Alliés occidentaux. Quoi qu'il en soit, la conception actuelle est incomparablement plus conforme que l'ancienne à la réalité historique.

Proportionnellement, c'est la période qui suivit 1989 qui pose le moins de problèmes. Après l'expérience d'une interprétation de l'histoire contemporaine pétrie d'idéologie communiste, c'est aujourd'hui une conception factuelle qui prévaut dans le traitement et la présentation de l'histoire tchèque et mondiale. Les auteurs des manuels sont en effet tout à fait conscients du fait que le laps de temps qui les sépare de cette période est trop court pour leur permettre de prendre le recul et la hauteur nécessaires.

\section{Une nation dans l'Europe}

Au vu de ce qui précède, il apparaît donc que l'appréciation des événements historiques des $\mathrm{XIX}^{\mathrm{e}}$ et $\mathrm{XX}^{\mathrm{e}}$ siècles dépend étroitement de la situation politique nouvelle. Cette constatation vaut naturellement aussi pour la période présente, tout particulièrement sur les questions se rapportant aux problèmes germano-tchèques, russo-tchèques et slovaco-tchèques non résolus. Les politiciens ont évidemment intérêt à ce que l'interprétation qui est donnée de l'histoire dans les milieux spécialisés et les livres scolaires ne desserve pas leurs objectifs immédiats, le premier d'entre eux étant l'entrée de notre pays dans l'Union européenne et l'OTAN.

Tandis que le ton nettement critique des publications historiques consacrées à l'histoire russe et soviétique ne provoque pas de réaction particulière, il en va tout autrement dans le cas des relations germano-tchèques, à propos desquelles les représentants politiques des deux États proclament la nécessité de « dépasser » le passé et de réconcilier les deux nations. Les efforts qu'ils déploient pour mettre en lumière l'époque de la collaboration germanotchèque et pour éviter de faire allusion à certains conflits teintés de nationalisme sont quelquefois comiques. Surtout quand quelque historien tchèque (ou allemand), humant l'atmosphère politique, « retouche » par exemple les sentiments antiallemands du mouvement hussite ou « enjambe » allégrement les positions germanophobes d'une partie de l'intelligentsia tchèque non catholique, aux $\mathrm{XVI}^{\mathrm{e}}$ et $\mathrm{XVII}^{\mathrm{e}}$ siècles ${ }^{10}$. L'aspect politique est particulièrement net dans l'interpréta-

9 L'interprétation de l'histoire tchèque est fondée sur les points de vue représentés par les travaux très documentés rédigés par Karel Kaplan, dont Pravda o Československu (La Vérité sur la Tchécoslovaquie), Prague, 1990, et Nekrvavá revoluce (La Révolution non sanglante), Prague, 1993.

10 Pour la partie allemande, voir par exemple l'ouvrage de l'éminent spécialiste de l'histoire tchèque Ferdinand Seibt, Deutschland une die Teschen. Geschichte einer Nachbarschaft in der Mitte Europas, München, 1993, p. 156190. 
tion de l'histoire récente, par exemple dans l'appréciation des causes et des conséquences des accords de Munich (conclus en septembre 1938), dans l'analyse des années 1939-1945 et dans la présentation de l'expulsion des Allemands des Sudètes hors de la Tchécoslovaquie renaissante. Le devoir de la commission d'historiens tchéco-allemande, constituée en 1990, ne se réduisait pas à évaluer et à porter un jugement sur les rapports germano-tchèques aux $\mathrm{XIX}^{\mathrm{e}}$ et $\mathrm{XX}^{\mathrm{e}}$ siècles; elle était également censée remplir une mission politique cachée. Les conclusions des historiens devaient en effet cautionner le texte de la déclaration politique tchéco-allemande (dont l'approbation est prévue pour fin 1996) ; en cas d'échec, elles devaient apporter la preuve qu'une vision commune existe bel et bien, tout au moins au niveau des spécialistes ${ }^{11}$. Les membres de la commission ont beau jurer que leur rapport définitif ne cherche ni à influencer l'opinion publique ni à imposer une présentation "engagée » de l'histoire récente, il est difficile de les croire tout à fait; en effet, il est prévu de procéder à un très fort tirage de ce rapport.

Au ministère de l'Éducation, de la Jeunesse et des Sports également, certains fonctionnaires sont convaincus que l'on peut écrire l'histoire sur la base d'un consensus qui harmoniserait des avis à l'origine différents. Le désaccord du public spécialisé avec le ton militant de l'un des auteurs catholiques ${ }^{12}$, les protestations de la communauté juive à propos du titre de ce livre et son insatisfaction face à la faible place accordée à la problématique juive dans les manuels d'histoire scolaires ont obligé les fonctionnaires à créer une commission d'historiens chargée de surveiller le contenu des publications destinées à l'enseignement de l'histoire dans les écoles primaires et secondaires. Les tentatives méritoires faites pour limiter la diffusion des opinions extrêmes courent cependant le risque de se transformer en une vérification systématique de l'équilibre du commentaire. D'autres minorités (tsigane, allemande, ruthène, homosexuelle, etc.) pourraient subitement exiger d'être convenablement représentées dans les livres scolaires. Satisfaire tout le monde signifierait déraper vers l'extrême et renoncer à l'histoire, dont la tâche principale est d'appréhender les faits importants et leur contexte. Les historiens et les enseignants tchèques sont extrêmement sensibles à ce type d'interventions, car ils ont encore en mémoire l'époque où l'histoire des $\mathrm{XIX}^{\mathrm{e}}$ et $\mathrm{XX}^{\mathrm{e}}$ siècles se métamorphosait, sur ordre des administrations dirigeantes, en histoire quasi exclusive du mouvement ouvrier.

Dans l'immédiat, un tel danger peut être écarté. Le climat libéral qui a envahi après 1989 toutes les sphères de la vie a également touché le domaine éducatif, ainsi que la conception de l'histoire scolaire dans les différents types d'établissements nationaux (d’État), privés ou religieux. La variété de l'offre des

11 Voir Konfliktgemeinschaft, Katastrophe, Entspannung. Skizze einer Darstellung der deutsch-tschechischen Geschichte seit dem 19. Jahrhundert, München, 1996.

12 Pavel K. Mráček, Přiručka cirkevnich dějin (Le Manuel de l'histoire religieuse), Prague, 1995. 
programmes d'instruction et des manuels scolaires, ainsi que la relative souplesse des plans et des programmes scolaires laissent aux enseignants une grande liberté de conception et de présentation de l'histoire. Le ministère de l'Éducation veille seulement à ce que l'enseignement de cette matière initie les élèves des écoles primaires et secondaires " au respect de l'héritage culturel de l'humanité et de leur propre nation", les "sensibilise aux particularités ethniques, religieuses et culturelles des autres nations», leur apprenne "à les respecter " et "à prendre conscience de l'enrichissement que représentent pour le monde culturel actuel ces spécificités nationales ${ }^{13} \ldots$ ». Il est évident que cette perception trouve son origine dans le système de valeurs européen (ou plutôt euro-atlantique) que nous connaissons aujourd'hui, ce qui ne l'empêche pas de respecter tout autant les expériences historiques et les traditions de la société tchèque.

Pour ce faire, l'enseignement de l'histoire met davantage l'accent sur les connexions existant entre l'histoire nationale et européenne, voire mondiale, et sur leurs conséquences, et ce du haut Moyen Age à aujourd'hui. Le manuel actuellement utilisé dans les pays de l'Union européenne en est un bon exemple, qui convient aussi bien aux contextes anglais, français, italien qu'allemand. Pourtant, ces textes ne sont pas transposables en l'état à la réalité tchèque, ni même véritablement utilisables, étant donné qu'ils ne parlent que très peu des problèmes des nations (ethnies) minoritaires d'Europe centrale et orientale. De tout temps, en effet, le premier contact de l'homme avec l'histoire s'est effectué par l'intermédiaire de sa patrie (prise au sens étroit ou plus large), à laquelle le lie un rapport sentimental. Dans ces circonstances, je ne dissimulerai pas mon scepticisme face à un éventuel ouvrage scolaire qui rédigerait et présenterait une histoire englobant tout le territoire de la future Europe unifiée. Il est même impossible d'élaborer «le texte idéal " c'est-à-dire qui conviendrait à tout un chacun et prendrait en considération les diverses expériences historiques. Il serait plus une forme d'éducation destinée à européaniser qu'un livre d'histoire.

La grande majorité des manuels d'histoire édités en République tchèque après novembre 1989 sont des publications qui s'intéressent principalement à l'histoire de l'État tchèque et tchécoslovaque. Ce boom de l'édition est la réponse à une formidable demande en livres dépourvus de toute interprétation communiste. Certains éditeurs « traduisirent » cet intérêt à leur manière et proposèrent quasi immédiatement les textes des ouvrages utilisés pendant la Première République, soit entre 1918 et 1938, ou entre 1945 et 1948, c'est-à-dire avant l'arrivée au pouvoir des communistes ${ }^{14}$. Mais on se rendit rapidement à

13 Histoire. Manuels scolaires de la sixième à la neuvième année de scolarité. Programme d'enseignement "Základní škola » (Écoles primaires et collèges), Prague, 1996, page 5.

14 Voir par exemple Josef Pekar, Dějiny československé (Histoire tchécoslovaque), Prague, 1991 ; Zdenĕk Kalista, Stručne dějiny československé (Brève histoire tchécoslovaque), Prague, 1992. 
l'évidence : ces manuels, y compris l'œuvre classique de Josef Pekar̆, n'avaient plus guère qu'une valeur documentaire. Leur style archaïque, leur contenu - que les nouvelles acquisitions des historiens rendaient obsolète - et le fait qu'ils n'allaient pas au-delà de 1945 gênaient professeurs et élèves.

Les maisons d'édition s'adressèrent donc aux plus fameux historiens des écoles supérieures et des instituts académiques et leur demandèrent d'écrire des manuels destinés à l'enseignement secondaire de l'histoire tchécoslovaque. De là, les travaux des spécialistes sur l'histoire du XIXe siècle et celui du professeur d'université Otto Urban sur le passé tchécoslovaque, de ses origines ${ }^{15}$ à... 1918 seulement. Parallèlement, un collectif de l'institut historique de l'Académie des sciences se chargea, sous la direction de Jaroslav Marek, de l'histoire tchèque jusqu'en 1989, par conséquent en considérant l'histoire slovaque dans le seul cadre de l'État commun ${ }^{16}$. Ces deux derniers livres dépassaient, par leur ampleur et leur retentissement, l'ouvrage en deux tomes intitulé Déjiny zemí Koruny české ${ }^{17}$, œuvre collective de spécialistes des plus jeunes générations qui anticipait la partition de la Tchécoslovaquie, renonçant de ce fait délibérément à traiter de la problématique slovaque. Cette circonstance, ajoutée au rejet du marxisme exprimé par les auteurs et au fait que ce livre peut être utilisé comme ouvrage scolaire, firent de ce travail la publication historique de l'après-1989 la plus appréciée (le tirage atteint déjà 164000 exemplaires). Parmi les livres d'histoire destinés aux écoles primaires, le sympathique ouvrage intitulé Lidé $v$ dejinách ${ }^{18}$ mérite d'être mentionné, qui s'intéresse plus particulièrement à la vie quotidienne et à la culture matérielle.

La rédaction des nouveaux manuels d'histoire mondiale, qui consacrent d'ailleurs certains chapitres au passé tchèque, a duré légèrement plus longtemps. Néanmoins, il semble que cette nouvelle conception, eu égard au processus d'unification européenne en cours, soit appelée à un plus grand avenir que le partage traditionnel entre histoires tchèque et mondiale. Le premier ouvrage d'enseignement secondaire complet de l'après-1989 n'a pourtant pas répondu aux attentes. Světové dějiny, de Vratislav Čapek, Jaroslav Pátek et Otto Zwettler ${ }^{19}$, supporte certes, du point de vue graphique, la comparaison avec les publications d'Europe occidentale, qui ont l'avantage d'accorder une large place

15 Otto Urban, České a slovenské dějiny do roku 1918 (Histoires tchèque et slovaque jusqu'en 1918), Prague, 1991.

16 Jaroslav Marek et coll., České a československé dějiny HI (Histoires tchèque et tchécoslovaque - HII), Prague, 1991.

17 Pavel Bělina, Petr Čornej et coll., Dějiny zemi Koruny české I-II, Prague, 1992. Version française abrégée intitulée "Histoire des pays tchèques ", Paris, 1995.

18 Voir par exemple Lydia Petráňová, Strědovek. Dejepis pro 2. stupen základni školy (Le Moyen Age. Version destinée aux collèges), Prague, 1996.

19 Vratislav Čapek - Jaroslav Pátek - Otto Zwettler, Svetové dějiny H-II (Histoire mondiale), Prague, 1993. 
à l'histoire des civilisations non européennes, mais l'explication de la matière même reste assez superficielle. De ce point de vue, la série publiée par la maison d'édition Prace et rédigée par des historiens de l'université Charles, le livre des éditions Scienta et la rigoureuse Dejiny evropské civilizace ${ }^{20}$ sont supérieurs. Il est impossible de citer ici les ouvrages destinés aux établissements primaires, car leur nombre ne cesse de croître.

Ainsi donc, l'offre en livres d'histoire post-révolutionnaires destinés aux écoles primaires et secondaires est riche et varié, à tel point que certains professeurs éprouvent parfois quelques difficultés à s'y retrouver. La libéralisation globale générée par novembre 1989 a eu une autre conséquence : le ministère de l'Éducation, de la Jeunesse et des Sports, qui doit donner son feu vert au classement de toute publication dans la catégorie des manuels scolaires, s'est trouvé littéralement submergé sous un flot de livres d'histoire scolaires et il lui fut impossible de les traiter avec toute l'attention nécessaire. C'est pourquoi l'appréciation du ministère ne fut pas toujours pertinente. Ces deux dernières années, cependant, la situation s'est notablement améliorée et tous les textes sont soumis à une lecture rigoureuse.

Il incombe également au ministère de l'Éducation, de la Jeunesse et des Sports d'approuver l'ensemble des programmes d'études de chaque établissement scolaire et de vérifier leur efficacité. Mais son intervention dans le domaine de l'enseignement se borne aux programmes. Le choix des ouvrages qui serviront de base à l'enseignement relève de la compétence du directeur de l'école primaire ou secondaire et des professeurs d'histoire. Aujourd'hui, en République tchèque, la responsabilité de l'enseignement de l'histoire, qui est de transmettre à la jeune génération les connaissances historiques fondamentales, de l'aider à s'orienter dans le système des valeurs et de participer à sa formation culturelle, repose essentiellement sur la compétence, la largeur d'esprit et l'action des enseignants.

20 Voir Petr Čornej - Pavel Bělina, Dějiny evropské civilizace H-I (Histoire de la civilisation européenne), Prague, 1995 ; aux éditions Scienta, voir le manuel d'histoire mondiale Středovek HII (Le Moyen Age HII), rédigé par Vratislav Vaniček, Zděnek Smetánka et Věra Hrochová, et l'ouvrage intitulé Novověk HII (Les Temps modernes HII), d'Antonín Kostlán, Zdeněk Hojda et Pavel Bělina, Prague, 1994 ; aux éditions Práce, Jan Souček, Dějiny pravěku a starověku (Histoire de I'Antiquité et du Moyen Age), Zdenĕk Benĕk, Dějiny středoveku (Histoire du Moyen Age), ainsi que Miroslav Hroch, Dějiny novověku (Histoire des temps modernes) et Jan Kuklíkovi, Dějiny 20. století (Histoire du $x x^{e}$ siècle). 